\title{
Surpression engendrée au distributeur par la rupture d'une conduite forcée
}

\section{Overpressure caused at the distributor by a break in a penstock}

\author{
PAR L. ESCANDE \\ MEMERE DE L'INSTITUT \\ INGÉNIEUR I.E.T.

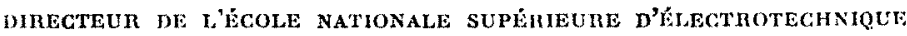 \\ ET D'HYDIRAULYUE DE TOULOUSE
}

\begin{abstract}
La rupture d'une conduite horizontale, en son milieu, provoque, an distributenr, une depression initiale, suivie de surpressions et dépressions alternées. La surpression obtenue, relativement modérée tant que la dechirure est insuffisante pour engendrer une cavitation atr distributeur, peut devenir très importante pour des ruptures plus étendues. La variation de la surpression maximum avec l'importance de la brèche présente de curieuses discontinuiles.
\end{abstract}

Considérons une conduite horizontale AB, ì caractéristique unique, alimentée par un réservoir, sous une pression de $y_{0}$ mètres d'eau (fig. 1). Nous supposons qu'une rupture, offrant au passage de l'eau une section utile $m s$, se produit instantanément, au milieu $c$ de la conduite : nous partons d'un état initial de repos, avec distributeur complètement fermé.

Nous négligeons la mise en vitesse et les per-

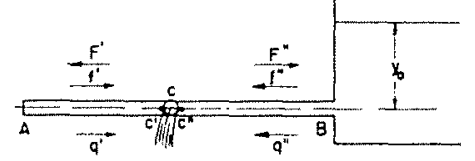

Fig. 1 .

tes de charge, ce qui est parfaitement admissible car nous nous limitons à des débits ne dépassant guère ceux qui interviennent dans Ic fonctionnement normal de l'usine.

Premier Cas. - Rupture assez faible pour ne. pas engendrer des cavitations au distributeur $A$.

Nous considérons que la breche $C$ est alimentée par les deux conduites $\mathrm{AC}^{\prime}$ (débit $q^{\prime}$ compté positivement de $\mathrm{A}$ vers $\mathrm{C}^{\prime}$ ) et $\mathrm{BC}^{\prime \prime}$ (débit $q^{\prime \prime}$ compté positivement de $B$ vers $C^{\prime \prime}$ ).

$q$ désignant le débit qui s'échappe de la brèche, a l'instant $t$, on a les relations :

$$
\xi=-y_{0}+\frac{q^{2}}{2 g m^{2} s^{2}}
$$

The rupture at the middle of a horizontal pipe first produces a depression at the distributor then alternate over-pressures and depressions. The over-pressure obtained is relatively small when the break is not big enough to cause cavitation at the distributor, but becomes very important for larger brealis. The variation of the maximum over-pressure with the size of the rupture presents curious discontinuities.

$$
q=q^{\prime}+q^{\prime \prime}
$$

Soit $P$ la parabole représentée par l'équation (1), dans le diagramme habituel en $(q, \xi)$ de la méthode de Bergeron (fig. 2), soit $n^{\prime}$ et $n^{\prime \prime}$ les

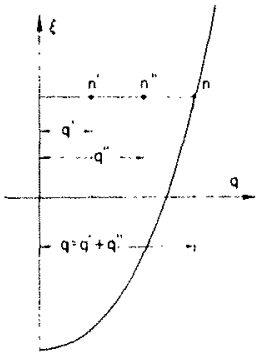

Fig. 2.

points de fonctionnement en $\mathrm{C}^{\prime}$ et $\mathrm{C}^{\prime \prime}$ au mêmo instant, la continuité des pressions exige que la surpression ait la même valeur en $C^{\prime}$ et $C^{\prime \prime}$; il en résulte que les points $n^{\prime}$ et $n^{\prime \prime}$ sonl situés sur une même horizontale qui, d'après (2), doit couper la parabole $\mathrm{P}$ en un point $n$ d'abscisse égale ‘ $q^{\prime}+q^{\prime \prime}$.

Nous prenons comme unité de temps $\theta / 4$, en appelant $\theta$ la durée d'un aller-retour d'onde d'un bout à l'autre de la conduite $A B$, avec les valeurs suivantes, caractérisant l'installation étudiée :

la pression statique........ $y_{0}=50 \mathrm{~m}$

la section de la conduite.......S $\mathrm{S}=0,50 \mathrm{~m}$

la célérité des ondes......... $a=100 \times g$ 
I.- A. - Nous considérons, en premier lia'u, une brèche assez petite pour n'appeler dans la conduite, en régime permanent, qu'un debit tròs faible $q_{n}=0,10 \mathrm{~m} / \mathrm{s}$ :

$$
a v_{0}-=100 \times 0,20=20 \mathrm{~m}
$$

Avant la rupture. tous les points de fonctionnement sont groupés à l'origine des coordonnées $0(q=0, \quad \xi=0)$ (fig. 3). L'ouverture inslantanée de la brèche transporte les points de fonctionnement en $\mathrm{C}^{\prime}$ et $\mathrm{C}^{\prime \prime}$ sur les droites confondues $\varpi^{\prime}$ et $\varpi^{\prime \prime}$, issues de 0 , en deux points confondus 02', $12^{\prime \prime}$ tels que l'horizontale correspondante coupe la parabole en un point 02 d'abscisse double de celle de $02^{\prime}$ et $02^{\prime \prime}$.

Les ondes engendrees dans les deux tronçons de conduites, après réflexion en $A$ et $B$, ne reviemnent en $C$ qu'apres deux unités de lemps: cola justifie la notation adoptée pour les pounts de fonctionnement $02^{\prime}$ et $02^{\prime \prime}$, le premier et lo dernier chiffre correspondant respectivement aux instants marquant le début et la fin du fonclionnement en ce point.

Le point $13-$ A est situé sur l'axe des du point de fonctionnement en $A$, en l'absence de cavitation $(q=0)$ et sur la droite $\Phi$ ' issue de (12'; de mème 13-.. B est placé à l'intersection de l'axe des $q$, lieu du point de fonctionnement 'n $B(\xi=0)$, et de la droite $\Phi$ " issue de $02^{\prime \prime}$.

Les points 24' et '24" se trouvent respectivement sur les droites of of $\varphi^{\prime \prime}$ issues de $13-\mathrm{A}$ et
13- B et leurs positions, sur ces droites, s'obliennent sans difficulté par tâtonnements. l'horizontale 24' - 24" devant couper la parabole P' au point 24 ayant comme abscisse la somme algébrique de celles de $24^{\prime}$ et 24 ".

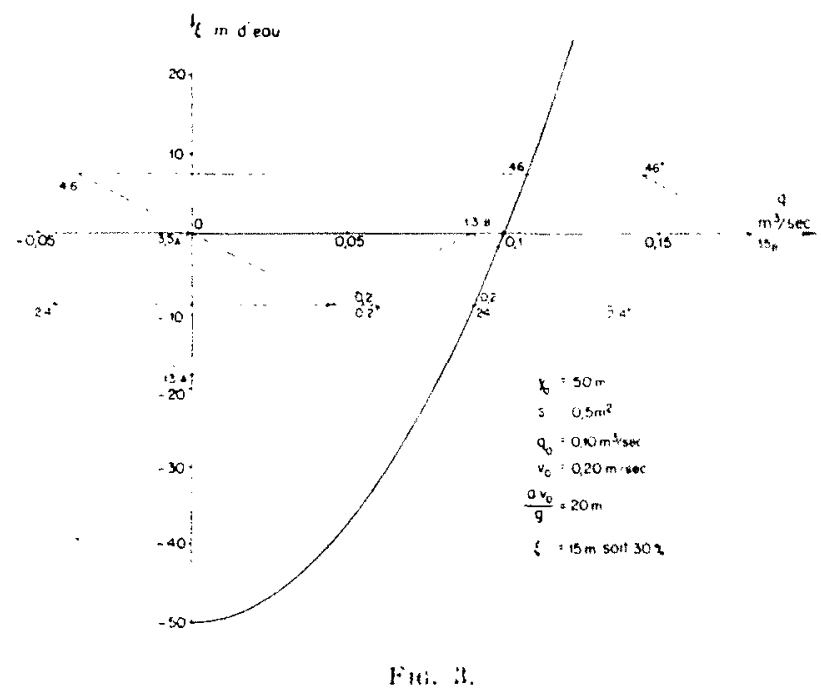

Des points $24^{\prime}$ el $24^{\prime \prime}$, on déduit $35-$ - A sur $(1 \vdots$ confondu avec 0 ) el 35 - B sur 0 q, el la construction se poursuit ainsi, de proche en proche (fig. 4).

La figure 5 donnc les courbes des surpressions en fonction du temps, at distributeur $A$ et à lit hroche $C$.

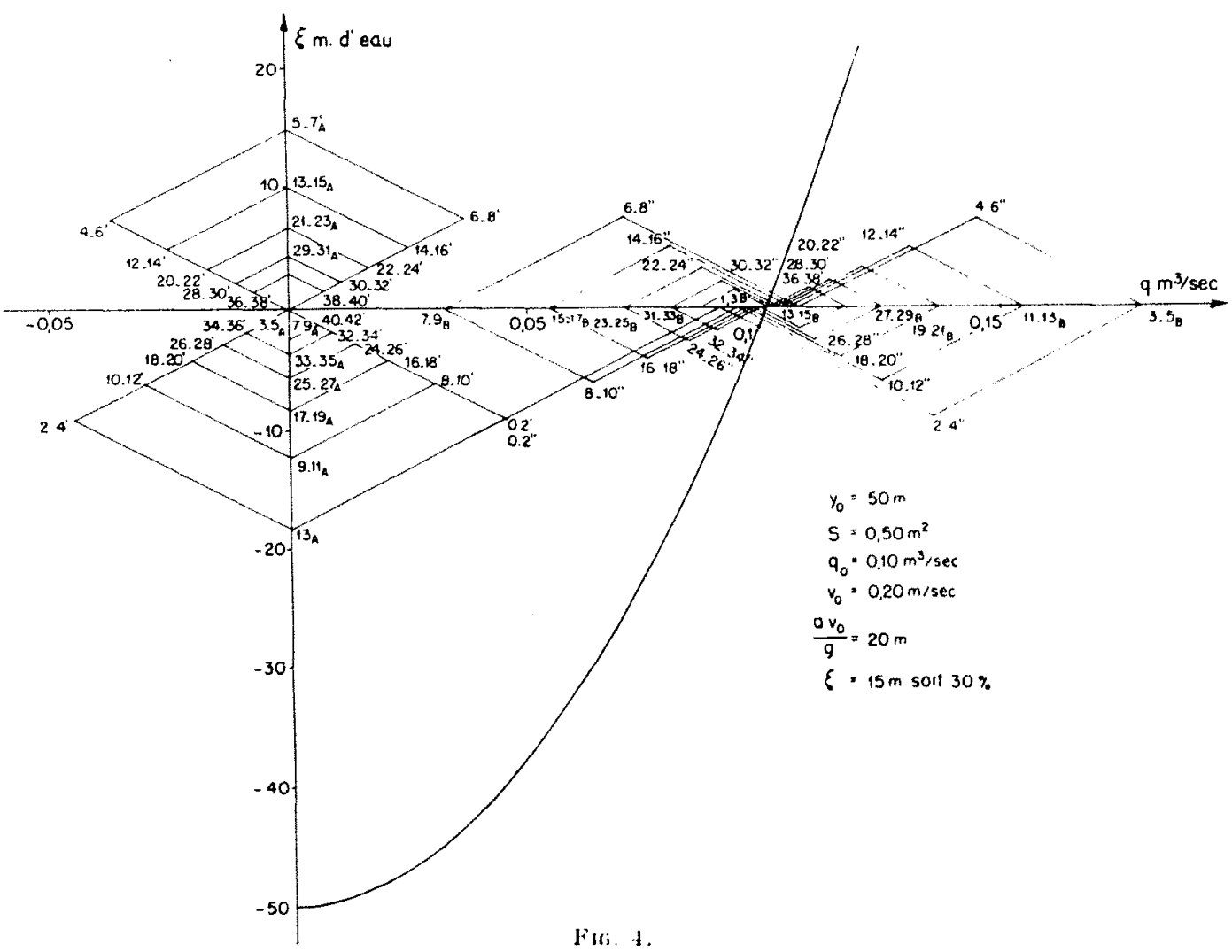


I - B, - - Nous avons appliqué la mème méthode à l'étude de brèches d'importance croissante, jusqu’à la valeur limite au-dessus de laquelle une cavité se forme en $A$, cas que nous cxaminons dans la seconde partie de cette ètude.

Nous arons caractérisé chacun des exemples

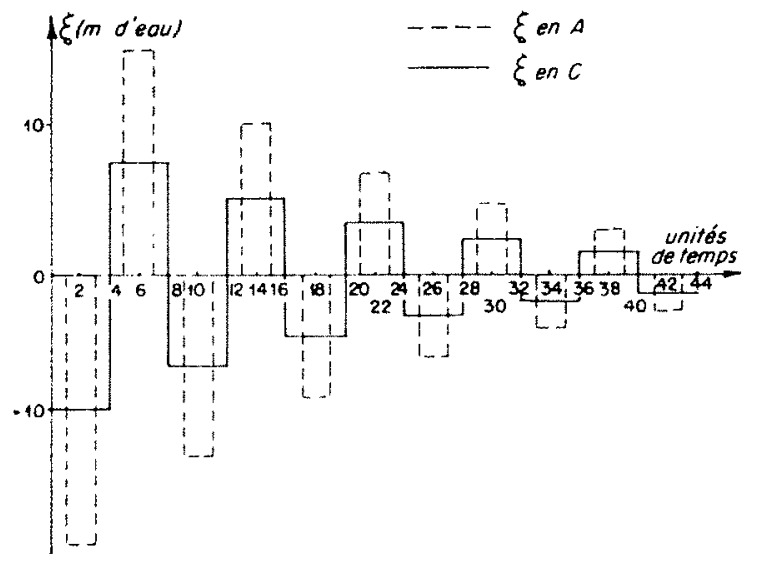

Fici. 5.

ainsi étudiés par la valeur du débil $\psi_{0}$ de régime permanent s'écoulant par la brèche.

Le tableau ci-après donne, en fonction de $q_{0}$, les valeurs de la dépression maximum $\breve{\zeta}_{\mathrm{AS}} \mathrm{el}$ de la pression maximum $\xi_{M}$ atteintes au distributeur $A$, ainsi que celles du quotient $\xi_{\mathrm{N}} / y_{0}$. Pour simplifier, nous avons admis que la pression atmosphérique correspond à $y_{n}=10 \mathrm{n}$.

\begin{tabular}{|c|c|c|c|}
\hline$q_{9} \mathrm{~m}: \% / \mathrm{s}$ & $\xi_{3} m$ & $\xi_{\mathrm{N}} m$ & $\xi_{\mathrm{N}} / y_{0}$ \\
\hdashline$\ldots, 100$ & $-18,2$ & 15,00 & 0,30 \\
\hline 0,200 & $-32,6$ & 21,00 & 0,42 \\
\hline 0,270 & $-11,2$ & 22,40 & 0,4475 \\
\hline 0,357 & -50 & 21,50 & 0,43 \\
\hline 0,474 & -60 & 17,20 & 0,344 \\
\hline
\end{tabular}

On voit que la dépression croit sans arrêt avec l'importance de la brèche, tandis que la surpression en A augmente tout d'abord puis décroît après ètre passée par un maximum.

Deuxième cas. - Rupture assez importante pour engendrer une cavitation au distributeur $A$.

En A, la dépression ne peut devenir supérieure a $y_{0}+y_{a}=60 \mathrm{~m}$. De plus, le débit n'est pas forcément nul en $A$, pendant la cavitation; un débit positif est toujours possible, avee formation d'une cavité, un débit négatif pouvant lui-même intervenir, quand une eavité préexiste, jusqu’à complet remplissage de celle-ci.

Done, tant qu'une cavité existe en $A$, le lieu du point de fonctionnement en $A$ est la droite $\Delta \quad(\xi=-60 \mathrm{~m})$ correspondant au vide en $\mathrm{A}$ : quand la cavité est refermée, le lieu du point de fonctionnement redevient l'axe $q=0$.

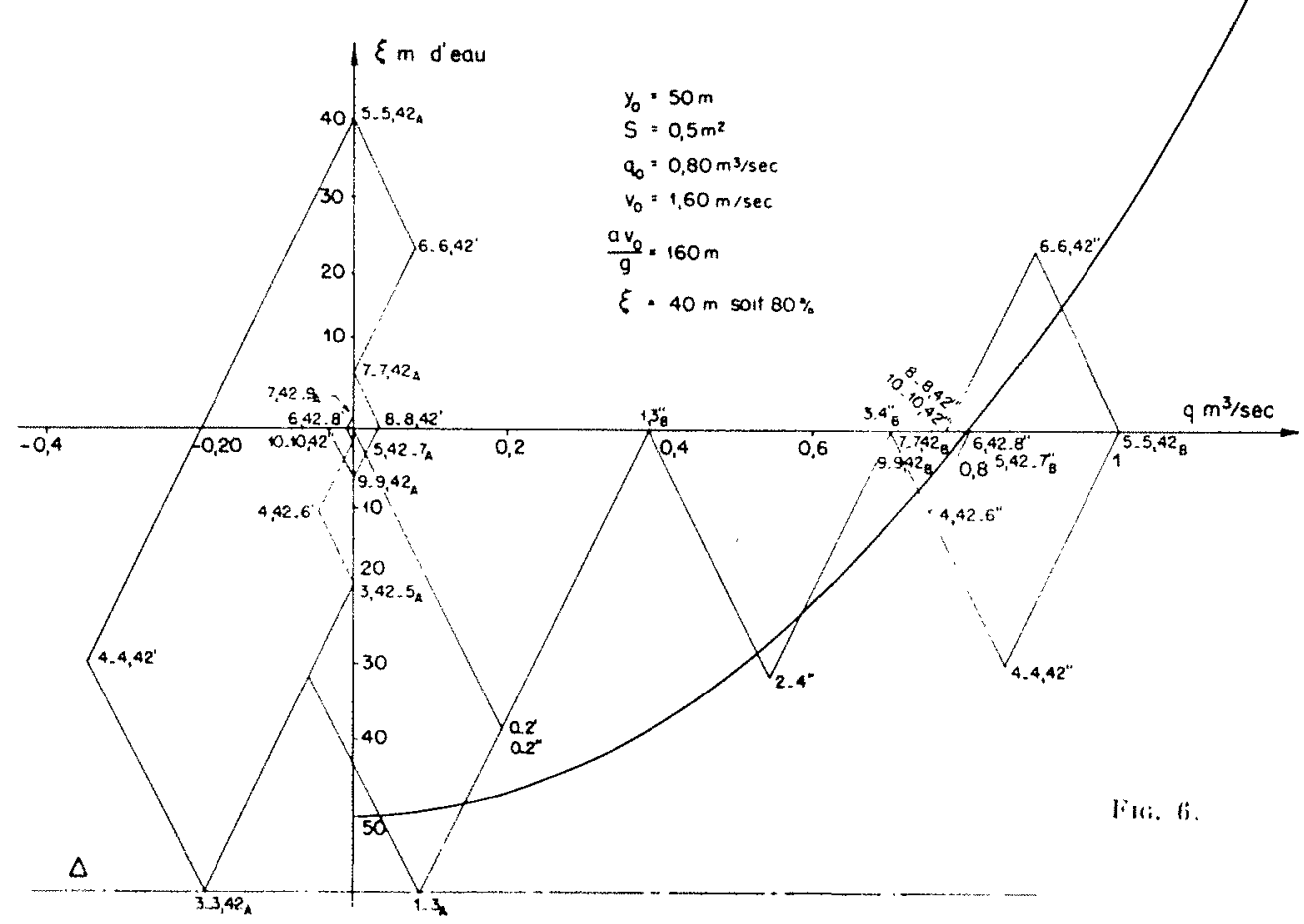


II -- A. - Considérons, par exemple, te graphique relatif à $q_{0}=0,80 \mathrm{~m}^{3} / \mathrm{s}$ (fig. 6 ).

Le point d'intersection de l'axe $0 \xi$ et de la droite $\Phi^{\prime}$ issue de 02', ne peut être retenu car il se place au-dessous de la droite $\Delta$ : le point de fonctionnement 13 -.. A se trouve done sur $\perp$ et une cavite se forme, durant deux unités de lamps, avec un débit de $0,084 \mathrm{~m}: \mathrm{s}$.

Les points $13-\mathrm{B}, 24$ " et $24^{\prime}$ étant dólerminés selon les principes déjà exposés, cherchons l: position du ou des points de fonclionnement on A pendant l'intervalle de temps 35 .

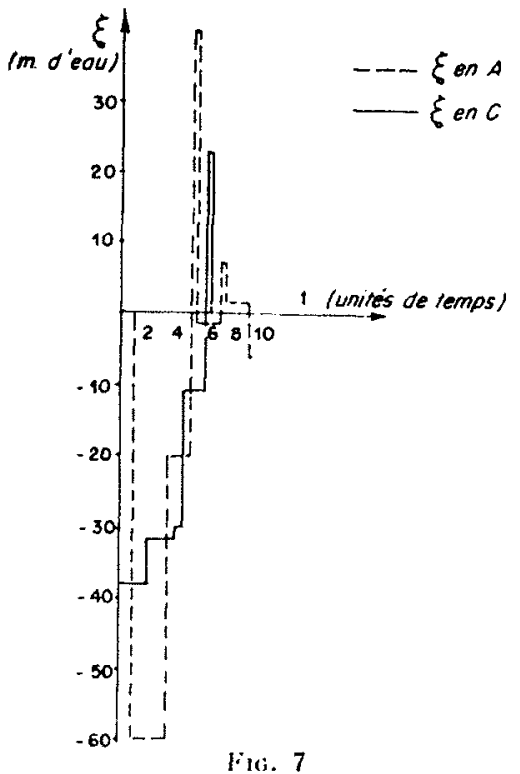

Au début de cel intervalle, le point est en $3.3,42$ - A à l'intersection de la droite $\Delta$ et de la droite ' 1 ' avec un dibit négatif $0,40 \mathrm{~m}^{*} / \mathrm{s}$, en un temps çgal $\dot{a}$ :

$$
\frac{2 \times 0,084}{0,40}=0,42
$$

Ensuile, pendant l'intervalle de temps 3,42.5, la cavité itant fermóe, le point de fonctionnement saute en $3,42.5 \cdots A$, à l'intersection de $0 \stackrel{3}{3}$ et de la droite 'D' issue de 24'. Lal construction habituelle eontinue ensuite, mais, dans chaque intervalle de deux unites de temps consécutives, il y a maintenant deux points de fonclionnement successifs à considérer en $\mathrm{A}, \mathrm{B}, \mathrm{C}^{\prime}$ et $\mathrm{C}^{\prime \prime}$.

La figure 7 donne les courbes $(i, \xi)$ en $A$ et $C$.

II -..- B. - Nous avons aussi étudié les cas relatifs aux valeurs de $q_{0}$ indiquées dans le tableau suivant, qui fournit également les valeurs de la surpression maximum $\xi_{M}$ en $A$, ainsi qua celles du rapport $\xi_{x} / y_{0}$.

\begin{tabular}{|c|c|c|}
\hline$q \mathrm{~m}^{3} / \mathrm{s}$ & $\xi_{M} m$ & $0_{\mathrm{Mr}} / y_{0}$ \\
\hline 0.474 & 60 & 1,20 \\
\hline 0,5 & 58,2 & 1,165 \\
\hline 0,8 & 10 & 0,80 \\
\hline 1,0 & 28,2 & 0,565 \\
\hline 1.1 & $\begin{array}{l}21,6 \\
80\end{array}$ & $\begin{array}{l}0,43 \\
1,60\end{array}$ \\
\hline 1.2 & 72.10 & 1,15 \\
\hline 1.5 & 57,00 & $1,1.1$ \\
\hline 2 & 31 & 0,68 \\
\hline 2,2 & $\begin{array}{l}27 \\
80\end{array}$ & $\begin{array}{l}0,54 \\
1,60\end{array}$ \\
\hline $2, \overline{3}$ & 68 & 1.36 \\
\hline
\end{tabular}

On observe la forme de la combe domant $\xi_{x} / q_{0}$ en fonction de $q_{0}$ (fig. 8 ), curieuse par ses discontinuités autres que celle, a priori normale. qui marque lapparition des cavitations.

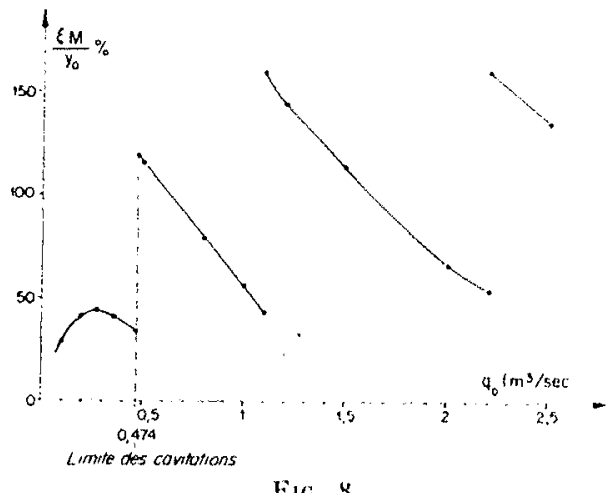

$F_{10}, 8$

DISCUSSION

II. le Président (janel remercie M. Hscasue et souligne l'intérét de la méthode graphique Bergeron; lors des expériences faites a Toulouse par MM. Camber et Eydoux, et auxquellos M. GahibL a participé et associé son nom, l'étude de phénomènes aussi compliqués et discontinus que eelui qui vient d'ête decrit aurat paru inacessible, mais la methode Bergeron permet, maintenant, de les suive glaphiquement, et $\mathrm{N}$. Escasib: a pleinement réussi a les mettre en lumiere de cette facon.

II. Ailhener rapproche le phénomene hydranlique de la rupture d'une conduite de celui du comp de foudre sur une ligne clectrique : dans chaque phesomene it $y$ a reflexion avec changement de signe et avec anmulion soit d'une des grandeurs soit de l'autre. 